\title{
PROCESSAMENTO DE CARTUCHOS DE IMPRESSORAS DE JATO DE TINTA: UM EXEMPLO DE GESTÃO DE PRODUTO PÓS-CONSUMO
}

Felipe Pereira de Moura, Rafael da Silva Oliveira e Júlio Carlos Afonso*

Departamento de Química Analítica, Instituto de Química, Universidade Federal do Rio de Janeiro, Av. Athos da Silveira Ramos, 149, B1. A, 21941-909 Rio de Janeiro - RJ, Brasil

Cláudio Augusto Vianna e José Luiz Mantovano

Departamento de Química e Materiais Nucleares, Instituto de Engenharia Nuclear, Rua Hélio de Almeida, 75, 21941-906 Rio de Janeiro - RJ, Brasil

Recebido em 7/9/11; aceito em 23/1/12; publicado na web em 15/5/12

\begin{abstract}
PROCESSING OF INK-JET PRINTER CARTRIDGES: AN EXAMPLE OF MANAGEMENT OF A POST-CONSUMPTION PRODUCT. This work presents a route for processing spent ink-jet cartridges in an experimental course. The disassembly of the cartridges requires several steps and the recognition of their different components is essential to define the best final destination (recycling, co-processing). The plastic strips were chemically processed so as to recover gold and copper. The students recognized the difficulty of processing multicomponent wastes and the importance of the chemical work under the best safety conditions; they also experienced many laboratory techniques and recognized the value of the selective collection and the reverse logistics to reach a viable commercial scale recycling.
\end{abstract}

Keywords: waste electrical and electronic equipment (WEEE); waste management; ink-jet cartridge.

\section{INTRODUÇÃO}

Os equipamentos eletroeletrônicos (EEE) podem ser considerados uma maravilha da ciência, ${ }^{1}$ pois a junção dos mais diferentes materiais (plásticos, resinas, metais etc.) permite a concepção de produtos que revolucionam e interagem constantemente com o cotidiano do consumidor, face à extraordinária esfera de dinamismo em que o mundo globalizado de hoje está envolvido. Porém, o ritmo impressionante do crescimento da fabricação e do consumo de EEE, combinado à tendência à redução da vida útil das gerações dos mesmos (obsolescência programada), levam à produção do chamado lixo eletroeletrônico ou resíduos de equipamentos eletroeletrônicos (REEE), que assumiu proporções alarmantes em todo o mundo. Estimativas indicavam que, em 2010, haveria 600 milhões de computadores obsoletos nos EUA. Considerando o resto do mundo, esse número ultrapassaria 3 bilhões. ${ }^{2}$ Atualmente, cerca de 1,5 bilhão de celulares são substituídos por ano. ${ }^{3}$ A Organização das Nações Unidas (ONU) estima que a produção de lixo eletroeletrônico atingiu 150 milhões de t em 2010. ${ }^{4} \mathrm{Em} \mathrm{2008,}$ foram geradas 149,2 mil t de lixo eletroeletrônico no Brasil. ${ }^{5} \mathrm{O}$ lixo eletroeletrônico cresce a uma velocidade três a cinco vezes maior que a do lixo urbano. ${ }^{5}$

A produção cada vez maior e mais rápida de EEE traz dois grandes riscos ambientais: o elevado consumo de recursos naturais na fabricação destes e a destinação final inadequada. ${ }^{6}$ Dados de caracterização de diversos EEE indicam que neles podem existir substâncias cujos constituintes contenham até 60 elementos da Tabela Periódica. ${ }^{6-8}$ Encontram-se nos EEE tanto substâncias com constituintes tóxicos (mercúrio, chumbo, berílio etc.) como metais preciosos (prata, platina, ouro etc.), cada qual com sua finalidade específica. ${ }^{7,8}$ Por isso, o primeiro grande impacto do lixo eletroeletrônico não é o seu descarte, mas sim a extração dos insumos necessários à sua fabricação. Estima-se que cerca de um milhão de computadores sejam descartados no país por ano. ${ }^{9}$ A grande parte vai para terrenos baldios, lixões e outros destinos inadequados. A Lei 12305/2010 (Política Nacional

*e-mail: julio@iq.ufrj.br de Resíduos Sólidos) prevê nos artigos 30 a 36 (Capítulo III, Seção II) a responsabilidade compartilhada de fabricantes, importadores, distribuidores e vendedores na logística reversa para os seguintes produtos pós-consumo: pilhas, baterias, produtos eletroeletrônicos, lâmpadas, óleos lubrificantes, pneus e agrotóxicos. ${ }^{10}$

$\mathrm{O}$ ouro pode ser tomado como um exemplo de como a fabricação de EEE impacta o mercado global de uma matéria-prima. Ele é amplamente utilizado em dispositivos eletroeletrônicos devido à sua excelente condutividade, ductibilidade e resistência à corrosão. ${ }^{1}$ Achase presente em placas de circuito impresso (computadores, celulares, calculadoras etc.), cartuchos de tinta e associado ao cobre, como uma fina camada que recobre conectores para contatos elétricos. ${ }^{1} \mathrm{O}$ teor de ouro nos EEE varia muito de equipamento para equipamento e, para um dado produto, entre as diversas gerações do mesmo. Calcula-se que há 300-350 g do metal em $1 \mathrm{t}$ de placas de circuito impresso de telefones celulares e 200-250 g em $1 \mathrm{t}$ de placas-mãe de computadores, ${ }^{3}$ teores muito superiores àqueles encontrados em fontes naturais. ${ }^{3,11} \mathrm{~A}$ escassez das fontes naturais de ouro combinada ao seu uso crescente em EEE levam a considerar os REEE como fonte secundária do metal. Seu preço praticamente dobrou entre 2003 e $2008 ;{ }^{12}$ da produção mundial em 2010 (2.500 t), cerca de $300 t$ foram para fabricação de itens para EEE, ${ }^{13,14}$ com taxa de crescimento do consumo da ordem de $12 \%$ ao ano. As reservas mundiais conhecidas de ouro atingem $47 \mathrm{mil} \mathrm{t}$, o que garante o suprimento do metal por apenas cerca de 20 anos. . $^{13,14}$

Face à composição complexa dos EEE, as estratégias para se recuperar um metal ou componente dos mesmos dependem do produto a ser processado, porque isso determina o número de etapas necessárias para se atingir o objetivo desejado. ${ }^{14}$ De modo geral, o processamento do EEE mais simples fornece conhecimento para desenvolver soluções factíveis para os EEE de composição mais complexa. ${ }^{1,8} \mathrm{O}$ cartucho de impressora de jato de tinta ${ }^{15}$ é um exemplo em pequena escala do desafio de processar os EEE.

Outra solução, que na verdade adia o problema de gerir um REEE para o futuro, é o reuso dos EEE. O cartucho vazio é um exemplo notável dessa prática no Brasil. No país existem três tipos de cartuchos: 
os originais novos, produzidos pelos fabricantes das impressoras; os compatíveis, que são novos mas produzidos por outros fabricantes e, os remanufaturados, ${ }^{16}$ que são cartuchos originais vazios reutilizados, após um processo que inclui teste de seus circuitos eletrônicos, remoção dos vestígios da tinta anterior, substituição de peças, recarga e controle de qualidade de impressão. A diferença entre um cartucho remanufaturado e um recarregado é que a recarga é o simples fato de encher novamente o cartucho de tinta sem nenhum controle. ${ }^{17,18}$

Estima-se que o mercado nacional de recarga de cartuchos cresce a uma taxa de cerca de $30 \%$ ao ano. ${ }^{18}$ Segundo a ABRECI (Associação Brasileira de Recondicionadores de Cartuchos para Impressoras), $40 \%$ do total de cartuchos originais vendidos no mercado são recarregados. ${ }^{19} \mathrm{O}$ país é hoje um dos mercados mais avançados em termos de reuso de cartuchos e toners de impressoras do mundo; ${ }^{16,17}$ calcula-se que haja mais de 5 mil empresas que fazem a recarga de cartuchos e toners vazios, fora as que atuam informalmente. ${ }^{20,21} \mathrm{~A}$ importância da remanufatura no país deve-se ao crescimento do mercado de impressoras, algo em torno de $20 \%$ ao ano ${ }^{17,20,22}-7,2$ milhões de impressoras foram vendidas em 2010, sendo 3,5 milhões de jato de tinta; ao preço elevado dos produtos originais e, à existência de pessoal qualificado. A atividade de remanufatura de cartuchos deve observar a legislação ambiental de seu Estado no tocante à gestão de resíduos (tintas, óleos, graxas etc.) oriundos de seus processos. ${ }^{16,22} \mathrm{O}$ setor envolveu em 2010 mais de 50 mil empregos diretos e movimentou mais de $\mathrm{R} \$ 100$ milhões.

Um cartucho não pode ser indefinidamente recarregado, sendo recomendado um máximo de três ciclos. ${ }^{16,18,19} \mathrm{~A}$ impossibilidade de recarga é atestada quando se nota que pelo menos um dos circuitos que conduzem a tinta não responde ao estímulo elétrico de um aparelho de teste. No Brasil, o cartucho não recarregável é geralmente descartado junto com os resíduos sólidos urbanos. Esta prática é de elevado impacto ambiental e precisa de equacionamento, podendo ser considerada uma lacuna na gestão do produto no Brasil.

Em 2008, mais de 200 milhões de cartuchos foram descartados em aterros só nos Estados Unidos. ${ }^{23}$ Menos da metade dos cartuchos usados é reutilizada ou reciclada na Europa e nos Estados Unidos, segundo um estudo feito em 2007. ${ }^{24}$ Esse mesmo estudo indica que alguns fabricantes de impressoras perceberam que a remanufatura do cartucho é melhor do que a reciclagem como primeira opção.

O objetivo deste trabalho foi propor a alunos de um curso de graduação de Química uma prática experimental de processamento de cartuchos imprestáveis para reuso através da desmontagem dos mesmos, seguida de separação de cada um dos componentes, a fim de que se possa definir a melhor destinação para eles. Dada a natureza muito diferenciada desses componentes, com níveis de periculosidade distintos, um cuidado neste trabalho foi enfatizar a necessidade de adotar as melhores práticas de segurança e higiene ocupacional. A reciclagem, como qualquer outro processo que envolve o manuseio e o emprego de produtos químicos, exige um compromisso com a segurança. Este trabalho também é uma oportunidade para os alunos vivenciarem a dificuldade em gerenciar um resíduo multicomponente, do qual o lixo eletroeletrônico é um exemplo bastante marcante.

\section{PARTE EXPERIMENTAL}

\section{Filosofia do trabalho}

O modus operandi foi implementado a partir do $1^{\circ}$ período de 2011 em aulas práticas da disciplina Tratamento de Resíduos Químicos de Laboratório, eletiva para o curso de Química do Instituto de Química da UFRJ, ${ }^{25}$ sendo realizadas pelos alunos (em grupos de 4) sob a supervisão dos monitores e do professor responsável. Este trabalho é uma continuidade daquele anteriormente realizado com toda uma variedade de resíduos coletados em aulas experimentais para recuperação de compostos de diversos elementos. ${ }^{26}$

Os monitores pesquisaram e planejaram, com a ajuda do professor, as rotas de processamento dos cartuchos, sendo feitos os ajustes necessários para enquadramento no tempo de aula disponível (4 h). Uma semana antes da aula experimental, os alunos receberam um roteiro em que estudaram as fichas de informação de segurança de produtos químicos (FISPQs) dos produtos (pigmentos, solventes etc.) presentes nos cartuchos, ${ }^{27}$ para definir que equipamentos de proteção individual e coletiva deveriam ser empregados nos experimentos. Nesse mesmo roteiro havia a descrição das composições relatadas dos cartuchos, ${ }^{1,16,18}$ para planejar as rotas de desmontagem e segregação/ identificação dos componentes.

\section{Descrição e desmontagem dos cartuchos}

Foram empregados cartuchos de três fabricantes diferentes. Inicialmente, pesou-se cada cartucho em uma balança analítica. Em seguida, fez-se um teste de remoção manual (com o auxílio eventual de um estilete) das fitas plásticas contendo cobre e ouro, procurando classificar os cartuchos conforme a facilidade ou não de remoção dessas fitas. A seguir, os cartuchos foram colocados em um torno, na capela, onde se procedeu à abertura dos mesmos com o auxílio de ferramentas (serra, estilete, chave de fenda etc.). Os cartuchos foram classificados quanto à facilidade de desmonte e segregação de seus componentes. Estes últimos, incluindo as fitas, foram pesados em balança analítica. Esse procedimento permite aos alunos determinar a composição percentual dos cartuchos.

Os alunos usaram luvas ao longo de todo o procedimento supracitado.

\section{Processamento das fitas plásticas}

Metade das fitas foi picada com o auxílio de uma tesoura, de modo que o maior comprimento dos pedaços picados não excedesse $1 \mathrm{~mm}$. A outra metade foi mantida em sua forma original. Esta metade foi tratada com solução de $\mathrm{NaOH} 6 \mathrm{~mol} \mathrm{~L}^{-1}$, em capela, em placa aquecedora-agitadora $\left(10 \mathrm{~mL} \mathrm{~g}^{-1}, 50{ }^{\circ} \mathrm{C}, 200 \mathrm{rpm}, 1 \mathrm{~h}\right)$ para remoção da camada plástica que recobre as fitas. Em seguida, foram filtradas a vácuo em cadinho filtrante na capela e lavadas com água $\left(2 \mathrm{~mL} \mathrm{~g}^{-1}\right)$.

As fitas picadas ou tratadas com $\mathrm{NaOH}$ foram colocadas em béqueres de $50 \mathrm{~mL}$ sobre uma placa agitadora-aquecedora. Adicionaram-se $10 \mathrm{~mL}$ de água régia recém-preparada (para cada $1 \mathrm{~g}$ de fita) e um agitador magnético. A mistura reacional foi aquecida a $60{ }^{\circ} \mathrm{C}$ por um período de 1 a $2 \mathrm{~h}$, em capela. $\mathrm{O}$ volume foi mantido constante por reposição periódica de água destilada. Após o período estipulado, a mistura reacional foi resfriada e filtrada a vácuo em cadinho fitrante na capela. O resíduo insolúvel foi lavado com água $\left(2 \mathrm{~mL} \mathrm{~g}^{-1}\right)$ e as águas de lavagem foram juntadas ao filtrado. Esse resíduo foi seco com o auxílio de um secador e pesado em balança analítica.

A solução, de tonalidade verde turquesa, foi submetida à extração com éter etílico ou metilisobutilcetona a $25^{\circ} \mathrm{C}$, numa relação fase aquosa/fase orgânica (FA/FO) igual a 1, em um funil de separação, em capela. Após a separação das fases, o processo foi repetido mais uma vez com nova porção do solvente orgânico. Este foi, em seguida, removido a vácuo por meio de um evaporador rotatório (em capela) e o resíduo, amarelo, foi pesado. A fase aquosa residual foi evaporada a $5-10 \%$ do volume inicial também por meio de um evaporador rotatório (em capela) e resfriada em gelo, cristalizando uma massa verde clara, a qual foi separada por filtração em papel de filtro (em capela), lavada com éter etílico, seca em capela com o auxílio de 
um secador e imediatamente pesada, pois ela é higroscópica, sendo conservada em frasco fechado em dessecador.

\section{Métodos analíticos}

Os metais presentes nas soluções aquosa (antes e após a extração) e orgânica, bem como nos produtos finais sólidos obtidos, foram analisados por fluorescência de raios-x por dispersão de comprimento de onda (WDXRF). Os alunos pipetaram $200 \mu \mathrm{L}$ de solução ou colocaram $10 \mathrm{mg}$ de sólido em um papel de filtro de tamanho específico para o aparelho; o papel foi seco sob lâmpada incandescente $(300 \mathrm{~W})$ e, em seguida, coberto com filme de poliéster. Inicialmente, efetuaram-se análises qualitativas ("varredura") com as amostras. A seguir, foram estabelecidas curvas analíticas na faixa de 0,1 a $1,0 \mathrm{~g} \mathrm{~L}^{-1}$ de cobre ou

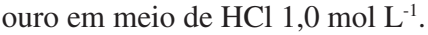

\section{RESULTADOS E DISCUSSÃO}

\section{Composição média dos cartuchos}

As composições dos cartuchos variaram pouco para cada modelo, conforme se observa na Tabela 1. Embora o sistema de armazenamento da tinta varie segundo o modelo, a composição geral de um cartucho - carcaça + sistema de tinta + fita plástica - não difere muito em todos os modelos avaliados. Os dados da Tabela 1 concordam com os dados da literatura. ${ }^{1,16} \mathrm{~A}$ principal variação se deve à quantidade residual de tinta presente nos cartuchos. A massa da fita depende do tamanho do cartucho, variando entre 0,4 e 1,6 g.

Tabela 1. Composição $(\% \mathrm{~m} / \mathrm{m})$ média dos cartuchos

\begin{tabular}{ccccc}
\hline Fabricante & $\begin{array}{c}\mathrm{N}^{\circ} \text { de } \\
\text { cartuchos }\end{array}$ & $\begin{array}{c}\text { Carcaça } \\
\text { plástica }\end{array}$ & $\begin{array}{c}\text { Sistema } \\
\text { de tinta* }\end{array}$ & Fita plástica \\
\hline A & 60 & $80-90$ & $10-17$ & $2-5$ \\
B & 24 & $65-69$ & $27-30$ & $4-5$ \\
C & 16 & $82-84$ & $10-12$ & $4-6$ \\
\hline
\end{tabular}

*Espuma (cartuchos A e B); lâmina de aço e bolsa plástica (cartucho C)

As carcaças foram examinadas quanto à identificação do polímero presente; $60 \%$ delas eram constituídas por PET (poli[tereftalato de etileno]) e $40 \%$ de uma mistura de PPE (polifenileno) + PS (poliestireno). A identificação se deu com base na sigla dos polímeros. De acordo com a Norma Brasileira NBR 13230, ${ }^{28}$ a mistura PPE + PS estaria na categoria 7 (“outros"), enquanto que o PET está na categoria 1. Esta identificação é de suma importância porque a reciclagem de materiais plásticos deve seguir alguns critérios, uma vez que certos polímeros não podem ser reciclados juntos. ${ }^{29}$

\section{Facilidade de desmonte do cartucho e de remoção das fitas plásticas}

A literatura indica que a remoção da fita plástica é um processo fácil. ${ }^{1}$ Entretanto, os dados da Tabela 2 indicam que isso não foi verificado nos cartuchos dos fornecedores B e C. No caso do fornecedor $\mathrm{B}$, em geral a fita saiu apenas parcialmente, enquanto que no caso das fitas do fornecedor $\mathrm{C}$, somente pequenos pedaços conseguiram ser removidos. Dentro da ótica de uma boa reciclabilidade dos produtos após o fim da vida útil, a impossibilidade de segregar a fita do cartucho inviabiliza a recuperação do cobre e do ouro. Esse é um aspecto que deveria ser levado em conta por todos os fabricantes de cartuchos dentro da premissa de conceber um produto que seja mais facilmente desmontável após o final de sua vida útil. ${ }^{30}$
Tabela 2. Facilidade de remoção da fita plástica contendo cobre e ouro do cartucho

\begin{tabular}{ccccc}
\hline Fabricante & $\begin{array}{c}\mathrm{N}^{\circ} \text { de } \\
\text { cartuchos }\end{array}$ & $\begin{array}{c}\text { Fita } \\
\text { inteiramente } \\
\text { removida }\end{array}$ & $\begin{array}{c}\text { Fita } \\
\text { parcialmente } \\
\text { removida }\end{array}$ & $\begin{array}{c}\text { Fita não } \\
\text { removida }\end{array}$ \\
\hline A & 60 & $54(90 \%)$ & $5(8,3 \%)$ & $1(1,7 \%)$ \\
B & 24 & $5(20,8 \%)$ & $15(62,5 \%)$ & $4(16,7 \%)$ \\
C & 16 & 0 & $5(31,3 \%)$ & $11(68,7 \%)$ \\
\hline
\end{tabular}

No que tange à desmontagem dos cartuchos, o comportamento foi totalmente diferente do observado para as fitas plásticas. Os cartuchos do fabricante $\mathrm{A}$ foram os mais difíceis de serem abertos, enquanto que os dos fabricantes $\mathrm{B}$ e $\mathrm{C}$ foram facilmente desmontados.

\section{Dissolução de ouro e cobre}

A água régia dissolve o ouro e o cobre segundo as reações:

$$
\begin{aligned}
& 3 \mathrm{Cu}+12 \mathrm{HCl}+2 \mathrm{HNO}_{3} \rightarrow 3\left[\mathrm{CuCl}_{4}\right]^{2-}+2 \mathrm{NO}+4 \mathrm{H}_{2} \mathrm{O}+6 \mathrm{H}^{+} \\
& \mathrm{Au}+4 \mathrm{HCl}+\mathrm{HNO}_{3} \rightarrow\left[\mathrm{AuCl}_{4}\right]^{-}+\mathrm{NO}+2 \mathrm{H}_{2} \mathrm{O}+\mathrm{H}^{+}
\end{aligned}
$$

$\mathrm{O} \mathrm{NO}$ é rapidamente convertido em $\mathrm{NO}_{2}$ (gás de coloração castanha) pelo oxigênio atmosférico. Após a evolução de todo o $\mathrm{NO}_{2}$ produzido, a solução adquiriu tonalidade verde clara relativa ao clorocomplexo de cobre (o do ouro tem coloração amarela brilhante).

A Tabela 3 mostra os resultados referentes ao efeito do tempo e do pré-tratamento na dissolução do ouro e do cobre para as fitas do fabricante A. O período necessário para dissolver a totalidade dos metais pela mistura ácida oxidante dependeu do procedimento prévio adotado. O pré-tratamento de lavagem com $\mathrm{NaOH}$ foi mais eficiente do que a picagem das fitas. Nesse último caso, cobre e ouro foram dissolvidos em mais de $99,5 \%$ após $2 \mathrm{~h}$, enquanto que no material pré-tratado com $\mathrm{NaOH}$ o mesmo resultado foi obtido após $1 \mathrm{~h}$. A diferença está na exposição da superfície dos metais ao agente lixiviante: o $\mathrm{NaOH}$ removeu o filme plástico externo, enquanto que a picagem não apresentou esse comportamento. Experimentos realizados à parte, na etapa de montagem da prática experimental, indicaram que um período de $3 \mathrm{~h}$ produziu o mesmo resultado que após $2 \mathrm{~h}$.

Tabela 3. Concentrações médias de ouro e cobre na fase aquosa $\left(\mathrm{mg} \mathrm{L}^{-1}\right)$ após digestão das fitas do fabricante $\mathrm{A}$ com água régia

\begin{tabular}{lccc}
\hline Pré-tratamento & Metal & $1 \mathrm{~h}$ & $2 \mathrm{~h}$ \\
\hline Solução de $\mathrm{NaOH} 6 \mathrm{~mol} \mathrm{~L}^{-1}\left(50^{\circ} \mathrm{C}, 1 \mathrm{~h}\right)$ & $\mathrm{Au}$ & 430 & 440 \\
& $\mathrm{Cu}$ & 11500 & 11610 \\
Picagem das fitas & $\mathrm{Au}$ & 370 & 440 \\
& $\mathrm{Cu}$ & 8890 & 11700 \\
\hline
\end{tabular}

Com base nos dados da Tabela 3, considerando-se $10 \mathrm{~mL}$ de água régia $\mathrm{g}^{-1}$ de fita, tem-se uma média de 4,4 mg de ouro e $116 \mathrm{mg}$ de cobre por cartucho. Esses resultados concordam com a literatura, que relata que um cartucho contém em média $125 \mathrm{mg}$ de cobre e $5 \mathrm{mg}$ de ouro. ${ }^{1}$

Além de cobre e ouro, a varredura simples das soluções aquosas ácidas por WDXRF acusou a presença de pequenas quantidades de ferro, alumínio e níquel em concentrações, respectivamente, da ordem de 10,50 e 120 vezes inferiores àquela do ouro mostrada na Tabela 3. 


\section{Recuperação de ouro e cobre}

Com base na discussão do item anterior, o processamento da solução aquosa ácida se resumiu à separação ouro-cobre. A extração do ouro por solventes orgânicos oxigenados é conhecida de longa data na literatura. ${ }^{31,32}$

A Tabela 4 mostra o desempenho da extração do ouro com éter etílico e metilisobutilcetona. Em um único estágio (FA/FO = $1 \mathrm{vol} /$ vol) à temperatura ambiente todo o ouro foi extraído por ambos os solventes, fato esse explicável pela baixa concentração do elemento nas soluções (Tabela 3). A fase orgânica tinha coloração amarela intensa, enquanto que a fase aquosa adquiria uma cor azul, referente aos íons $\mathrm{Cu}^{2+}$. Entretanto, o éter etílico foi menos seletivo do que a metilisobutilcetona. Isso se deve à solubilidade do éter etílico em água $(7,5 \% \mathrm{v} / \mathrm{v}$, e o éter dissolve $1,5 \% \mathrm{v} / \mathrm{v}$ de água, contra menos de $1 \mathrm{~g} \mathrm{~L}^{-1}$ no caso da cetona). ${ }^{33}$ Por isso, parte do cobre, ${ }^{31}$ alumínio, ferro e, mesmo, níquel foram coextraídos junto com o metal nobre. No caso da metilisobutilcetona apenas traços de ferro foram identificados.

Tabela 4. Desempenho da extração de ouro (FA/FO = 1 vol/vol, um estágio, $\left.25^{\circ} \mathrm{C}\right)$

\begin{tabular}{ccc}
\hline Metal & $\begin{array}{c}\text { \% extraído pelo } \\
\text { éter etílico }\end{array}$ & $\begin{array}{c}\text { \% extraído pela } \\
\text { metilisobutilcetona }\end{array}$ \\
\hline $\mathrm{Au}$ & 100 & 100 \\
$\mathrm{Cu}$ & 2 & 0 \\
$\mathrm{Fe}$ & 50 & 1 \\
\hline
\end{tabular}

Após a remoção do solvente orgânico por destilação a vácuo obteve-se um sólido amarelo, correspondendo ao $\mathrm{AuCl}_{3} \cdot \mathrm{H}_{2} \mathrm{O}$. Esse sólido contém em média $99,8 \% \mathrm{~m} / \mathrm{m}$ do ouro originalmente presente nas fitas. Não é aconselhável destilar à pressão atmosférica, porque o cloreto de ouro se decompõe com formação de sais básicos $\left(\mathrm{Au}(\mathrm{OH})_{x} \mathrm{Cl}_{3-x}\right)$, de coloração marrom-laranja, embora sejam solubilizados em solução diluída de $\mathrm{HCl}$, restabelecendo o íon complexo $\mathrm{AuCl}_{4} \cdot{ }^{-31} \mathrm{~A}$ evaporação da fase aquosa residual permitiu isolar o $\mathrm{CuCl}_{2} \cdot 4 \mathrm{H}_{2} \mathrm{O}$, o qual contém em média $99 \% \mathrm{~m} / \mathrm{m}$ de cobre solubilizado das fitas. As impurezas (Fe, Al, Ni) concentram-se na solução final.

\section{Vale a pena recuperar ouro e cobre de cartuchos?}

Individualmente, um cartucho contém muito pouco ouro, mas considerando-se o elevado consumo de cartuchos de impressoras do tipo jato de tinta no país, um sistema de coleta seletiva pode permitir o acúmulo de uma quantidade que viabilize a recuperação do ouro (e demais componentes dos cartuchos). Tomando como base o número de impressoras jato de tinta vendidas no Brasil em 2010 (3,5 milhões), teríamos um total de ouro circulante no país estimado em $3,5 \times 10^{6}$ unidades x $5 \mathrm{mg}$ Au por unidade ${ }^{1}=17,5 \times 10^{6} \mathrm{mg}$ $(17,5 \mathrm{~kg})$, e $3,5 \times 10^{6} \times 125=437,5 \times 10^{6} \mathrm{mg} \mathrm{de} \mathrm{Cu}(437,5 \mathrm{~kg})$, afora as impressoras adquiridas em anos anteriores e que continuam em funcionamento. Isso sugere que, com uma escala mínima adequada, pode-se recuperar ouro e cobre de cartuchos quando a recarga não for mais possível.

Os alunos foram estimulados a citar outros exemplos de resíduos multicomponentes em que é necessária uma escala considerável para permitir a reciclagem. As respostas mais comuns foram os EEE (teclado, mouse, impressora etc.), mas também foram citados: pilhas, baterias, lâmpadas fluorescentes, tomadas e interruptores elétricos.
Gestão dos componentes dos cartuchos e dos resíduos do processamento químico das fitas plásticas

Os solventes orgânicos destilados podem ser reutilizados para recuperar o ouro de novas porções de fitas tratadas com água régia, sendo apenas necessário repor as perdas por evaporação. As fitas picadas após tratamento com água régia (as quais não revelaram a presença de quaisquer metais de acordo com dados de WDXRF), bem como os resíduos de tinta, a esponja e a bolsa plástica podem ser coprocessados, em conformidade com a Resolução 264/99 do Conselho Nacional de Meio Ambiente (CONAMA). ${ }^{34} \mathrm{~A}$ solução ácida após o isolamento do cloreto $\mathrm{CuCl}_{2} \cdot 4 \mathrm{H}_{2} \mathrm{O}$ pode se neutralizada com a solução de $\mathrm{NaOH}$ usada no pré-tratamento das fitas, precipitando os metais presentes, ou então reaproveitada para compor a solução ácida para o tratamento de novas fitas. As carcaças plásticas e as lâminas de aço podem ser recicladas.

\section{CONCLUSÕES}

O processamento de cartuchos de impressoras de jato de tinta mostrou aos alunos a complexidade da gestão de um resíduo multicomponente, e que a concepção de produtos mais facilmente recicláveis após o fim da vida útil deve ser uma prática a ser adotada por todos os fabricantes de quaisquer produtos com essas características. A desmontagem e a identificação da natureza de cada componente é requisito fundamental para uma gestão bem sucedida dos mesmos. A reciclagem dos cartuchos pode ser uma solução ambiental quando seu reuso (remanufatura) não for mais possível, mas isso depende de uma logística reversa eficaz de coleta desses produtos.

No isolamento do ouro e do cobre, foi necessária uma escolha criteriosa de condições que permitissem maximizar a solubilização dos mesmos. A picagem das fitas deu piores resultados do que a remoção da camada polimérica com solução de $\mathrm{NaOH}$, pois esta expôs melhor a superfície dos metais ao agente lixiviante. O ouro foi extraído com menor interferência pelo solvente que tinha a menor solubilidade em água (metilisobutilcetona). A partir das soluções orgânica $(\mathrm{Au})$ e aquosa $(\mathrm{Cu})$ foi possível obter produtos finais com grau de pureza bastante bom.

O trabalho reforça as experiências anteriores, ${ }^{25,26}$ nas quais os alunos consolidam a sua formação em Química baseada na experimentação e no desenvolvimento de um espírito crítico através da discussão das melhores rotas de processamento de um resíduo multicomponente. A importância da higiene e da saúde ocupacionais na formação profissional do químico foi o outro ponto positivo, assinalado pelos próprios alunos.

\section{AGRADECIMENTOS}

Ao CNPq pelo auxílio financeiro. F. P. Moura e R. S. Oliveira agradecem ao Programa Institucional de Bolsas de Iniciação Científica (PIBIC/UFRJ-CNPq) a concessão de bolsas de iniciação científica.

\section{REFERÊNCIAS E NOTAS}

1. Serpe. A.; Artizzu, F.; Mercuri, M. L.; Pilia, L.; Deplano, P.; Coord. Chem. Rev. 2008, 252, 1200.

2. Frota Neto, J. Q.; Ruwaard, J. M. B.; van Nunen, J. A. E.; Heck, E. V.; Int. J. Prod. Econ. 2008, 111, 195.

3. Hagelüken, C.; Corti, C. W.; Gold Bul. 2010, 43, 209.

4. Wagner, T. P.; Waste Manage. 2009, 29, 3014.

5. Viktor, M.; Revista Galileu, n. 218, julho 2009, seção ambiente.

6. Oguchi, M.; Sakanakuma, H.; Terazono, A.; Takigami, H.; Waste Manage. 2012, 32, 96. 
7. Artoni, C.; Revista Galileu, n. 187, fevereiro 2007, seção ambiente.

8. Computers and the Environment: Understanding and Managing Their Impacts; The United Nations University: Amsterdam, 2004.

9. Spitzcovsky, D.; Planeta Sustentável, São Paulo, edição de 8 junho 2009.

10. Lei Federal 12.305/10 (Política Nacional de Resíduos Sólidos), 02/08/2010, Diário Oficial da União, 03/08/2010.

11. Ogunseitan, O. A.; Schoenung, J. M.; Sapheres, J. D. M.; Shapiro, A. A.; Science 2009, 326, 670

12. Manhant, A.; J. Ind. Ecol. 2010, 15, 13.

13. Ongondo, F. O.; Williams, I. D.; Cherrett, T. J.; Waste Manage. 2011, 31,714 .

14. Lim, S.; Kang, D.; Ogunseitan, O. A.; Schoenung, J. M.; Environ. Sci. Technol. 2011, 45, 320.

15. http://www.ebah.com.br/impressoras-pdf-a74361.html, acessada em Fevereiro 2011.

16. Idéias de Negócios - Recarga de Cartuchos, SEBRAE: Brasília, 2008.

17. Costa Filho, C. F. F.; Coelho Jr., L. C. B.; Costa, M. G. F.; Produção 2006, 16,100 .

18. http://www.sebrae-sc.com.br/ideais/default.asp?vcdtexto=3187, acessada em Março 2011

19. Moreira, D.; IDG NOW!, edição de 5 de dezembro de 2010, Seção Mercado, P \& D.

20. http://www.fourcolorsfortaleza.com.br/índex.php?option+com_conten t\&view+category\&layout=log\&id=1\&Itemid=76, acessada em Março 2011.

21. Chi, X.; Streicher-Porte, M.; Wang, M. Y. L.; Reuter, M. A.; Waste Manage. 2011, 31, 731 .
22. http://www.abreci.org.br/noticias/ler/34/, acessada em Março 2011.

23. The US Environmental Protection Agency, National Emission Standards for Hazardous Air Pollutants: Area Source Standards for Paints and Allied, Products Manufacturing; Federal Register 2009, 74, 26142.

24. 2007 Supplies Recycling: U.S. and Europe, InfoTrends: Bedfordshire, 2007.

25. Leite, Z. T. C.; Alcantara, S.; Afonso, J. C.; Quim. Nova 2008, 31, 1892.

26. Borges, B. G. A. L.; Silva, C. N.; Guedes, L. K. S.; Afonso, J. C.; Quim. Nova 2011, 34, 720 .

27. Os compostos citados nas composições das tintas dos cartuchos foram: 1,5-pentanodiol, 1,4-butanodiol, cetonas alifáticas (butanona, 2-pentanona), ésteres alifáticos (como acetato de etila e acetato de n-butila) e alcoóis (1-propanol, 2-propanol, etanol).

28. Associação Brasileira de Normas Técnicas; NBR 13230, ABNT: Rio de Janeiro, 2008.

29. Cangemi, J. M.; Santos, A. M.; Claro Neto, S.; Quimíca Nova na Escola 2005, $\mathrm{n}^{\circ} 22,17$.

30. Farias, L. A.; Fávaro, D. I. T.; Quim. Nova 2011, 34, 1089.

31. Ohlweiler, O. A.; Química Analítica Quantitativa, $3^{\mathrm{a}}$ ed., Livros Técnicos e Científicos: Rio de Janeiro, 1981, vol. 1, p. 283-287.

32. Brooks, R. B.; Chatterjee, A. K.; Ryan, D. E.; Chem. Geol. 1981, 33, 163.

33. Allinger, N. L.; Cava, M. P.; de Jongh, D. C.; Johson, C. R.; Lebel, N. A.; Stevens, C. L.; Química Orgânica, 2a ed., Guanabara Dois: Rio de Janeiro, 1978, p. 54.

34. Resolução do Conselho Nacional de Meio Ambiente (CONAMA) $n^{\circ}$ 264, de 26/08/1999, Diário Oficial da União, 20/03/2000. 